\title{
Identification of biomarkers and candidate regulators for multiple myeloma under the knockout of AURKA
}

Hanming Gu ${ }^{1,2}$, Wei Wang ${ }^{3}$, Gongsheng Yuan ${ }^{4}$

${ }^{1}$ School of Electronic, Information and Electrical Engineering, Shanghai Jiao Tong University, Shanghai, China

${ }^{2}$ SHU-UTS SILC Business School, Shanghai University, Shanghai, China

${ }^{3}$ Shanghai Civil Aviation College, Shanghai, China

${ }^{4}$ Department of Physiology and Pathophysiology, School of Basic Medical Sciences, Fudan University, Shanghai, China

"Corresponding author: Dr. Gongsheng Yuan, Department of Physiology and Pathophysiology, School of Basic Medical Sciences, Fudan University, Shanghai, China gsyuan14@fudan.edu.cn 


\section{Abstract}

Multiple myeloma $(\mathrm{MM})$ is a plasma cell malignancy that is characterized by the overabundance of monoclonal paraprotein. Aurora kinase A (AURKA) was upregulated in patients with high-risk MM. AURKA inhibitors were used to inhibit MM cell proliferation by inducing cell apoptosis and injury. In our study, we aim to identify biological processes and pathways of MM cells under the knockout of AURKA (AURKA $\mathrm{KO}$ ) by using a bioinformatics method to elucidate their potential pathogenesis. The gene expression profiles of the GSE163589 dataset were originally produced by using the high-throughput BGISEQ-500 (Homo sapiens). The biological categories and pathways were analyzed by the Kyoto Encyclopedia of Genes and Genomes pathway (KEGG), Gene Ontology (GO), and Reactom enrichment. KEGG and GO results indicated the biological pathways related to the immune responses and cancer activities were mostly affected in the development of MM with AURKA KO. Moreover, we identified several genes including GNG5, UBE2D1, and BUB1B were involved in the regulation of cancer genesis. We further predicted novel regulators that had the ability to affect the progression of MM with AURKA KO based on the L1000fwd analysis. Therefore, this study provides further insights into the mechanism of MM under AURKA inhibitor treatments.

\section{Introduction}

Multiple myeloma (MM) is a common malignancy of terminally differentiated plasma cells ${ }^{1}$. MM cells are originated in the bone marrow, but they also reside in the peripheral blood and other organs ${ }^{2}$. MM accounts for about $1.7 \%$ of all malignancies in the US. The incidence of MM is higher in Americans but lower in Asian and Hispanic individuals ${ }^{1}$. MM is characterized by the secretion of monoclonal immunoglobulin proteins that are produced by pathologic plasma cells ${ }^{3}$. The clinical manifestations include monoclonal protein, malignant cells, end-organ damage (bone disease with lytic lesions, anaemia, renal insufficiency, and hypercalcarmia) ${ }^{4}$. MM cells are affected by the bone marrow microenvironment because of the adhesion of MM cells to extracellular-matrix proteins ${ }^{5}$. In addition, binding of $\mathrm{MM}$ cells to BM accessory cells induces secretion of cytokines, which further promotes tumor cell activation ${ }^{6}$. 
Aurora kinases were found to regulate cell-cycle checkpoints and some related molecules such as cyclins and cyclin-dependent kinases ${ }^{7}$. Aurora kinases localize in the centrosome and play important roles in cell division by regulating chromatid segregation in mitotic cells; moreover, loss of chromatid segregation leads to genetic instability and tumorigenesis $^{8}$. There are three members of the mitotic Aurora kinase family: Aurora-A, Aurora-B and Aurora-C kinases. Aurora-A localizes to centrosomes and associates with spindle microtubules proximal to the spindle poles during mitosis ${ }^{9}$. Enhanced expression of AURKA was reported in several cancers including laryngeal, breast cancers, and MM. Moreover, AURKA associated with cell proliferation, metastasis and chemoresistance $^{10}$. AURKA was recently reported as a crucial target of MM. Upregulation of AURKA is related to centrosome amplification and worse prognosis in $M^{11}$. Reports showed that inhibition of AURKA gene expression in MM cells by RNAi leads to apoptosis and cell death as well as inhibits G2/M cell-cycle progression in MM cell lines ${ }^{12}$. Thus, regulation of AURKA and related pathways may be a convincing strategy in MM therapy.

In this study, we investigated the effect of the knockout of AURKA (AURKA KO) in MM cells. We identified several DEGs, candidate inhibitors and the relevant biological processes of MM cells with AURKA KO utilizing comprehensive bioinformatics analyses. We performed the functional enrichment analysis and protein-protein interaction for finding significant gene nodes. These key genes and signaling pathways could be critical to therapeutic interventions of MM.

\section{Materials and Methods}

Data resources

The dataset GSE163589 was downloaded from the GEO database (http://www.ncbi.nlm.nih.gov/geo/). The data was produced by BGISEQ-500 (Homo sapiens), Tianjin Medical University, China. Bulk RNA-Seq analysis was performed using LP-1 that was infected by the virus that packages sgRNA targeting AURKA gene or the non-target control. 
The dataset GSE163589 containing LP-1 control cells and LP-1 AURKA KO cells samples was conducted by $R$ script $^{13,14}$. We used a classical t test to identify DEGs with $\mathrm{P}<.01$ and fold change $\geq 1.5$ as being statistically significant.

Gene functional analysis

Gene ontology (GO) analysis is a useful tool to develop a comprehensive model of biological systems and to provide a system for hierarchically classifying genes ${ }^{15,16}$. Kyoto Encyclopedia of Genes and Genomes (KEGG) database is a commonly used tool for understanding the biological system, which integrates functional information, biological pathways, and sequence similarity. We performed the GO and KEGG pathway analyses by utilizing the Database for Annotation, Visualization, and Integrated Discovery (DAVID) (http://david.ncifcrf.gov/) and Reactome. $\mathrm{P}<.05$ and gene counts $>10$ were considered statistically significant.

Module analysis

The Molecular Complex Detection (MCODE) of Cytoscape software was used to analyze the densely connected regions in protein-protein interaction (PPI) networks ${ }^{17}$. The significant modules and clusters were selected from the constructed PPI network using MCODE. The function and pathway enrichment analyses were performed by using Reactome, and $\mathrm{P}<.05$ was used as the cutoff criterion.

Reactome and L1000FDW analysis

We performed the Reactom pathway (https://reactome.org/) and L1000FDW (https://maayanlab.cloud/L1000FWD/) to obtain the visualization, interpretation and analysis of potential pathways. $\mathrm{P}<.05$ was considered statistically significant.

\section{Results}

\section{Identification of DEGs in myeloma cells with AURKA KO}

LP-1 cells were infected by the virus that packages sgRNA targeting AURKA gene (Aurora A-KO) or the non-target gene (Control). AURKA KO cells were harvested for gene expression profiling. To gain the insights on LP-1 cells with AURKA KO genes, the 
modular transcriptional signature of AURKA KO cells was compared to that of the Control cells. A total of 586 genes were identified to be differentially expressed in AURKA KO cells with the threshold of $P<0.05$. The top 10 up- and down-regulated genes for AURKA KO cells and control samples are list in table 1.

\section{KEGG analysis of DEGs in myeloma cells with AURKA KO}

To identify the biological roles and potential mechanisms of the DEGs from AURKA KO versus Control in LP-1 cells, we performed KEGG pathway enrichment analysis (Supplemental Table S1). KEGG pathway database (http://www.genome.jp/kegg/) is a collection of genes that are highly similar in sequence for understanding the molecular interaction, reaction and relation networks. Our study indicated the top ten enriched KEGG pathways including "Pathways in cancer", "Proteoglycans in cancer", "Influenza A", "Hippo signaling pathway", "Oxytocin signaling pathway", "Alzheimer's disease", "Cell cycle", "Lysosome", "Measles", and "Leukocyte transendothelial migration" (Figure 1).

\section{GO analysis of DEGs in myeloma cells with AURKA KO}

Gene ontology (GO) analysis is a useful tool for hierarchically classifying genes, which contains cellular components (CC), molecular functions (MF), and biological processes (BP). Here, we identified top ten cellular components including "extracellular exosome", "cytoplasm", "membrane", “cytoskeleton”, "centrosome”, “Golgi apparatus", "mitochondrion", "cytosol”, "intracellular ribonucleoprotein complex", and "mitochondrial inner membrane" (Figure 1). We then identified top ten biological processes: "transport", "cell cycle", "cell division", "response to drug", "Rho protein signal transduction”, "T cell proliferation", "positive regulation of monocyte chemotactic protein-1 production", "negative regulation of synaptic transmission, GABAergic", "positive regulation of binding", and "somatic recombination of immunoglobulin gene segments" (Figure 1). We identified top ten molecular functions: "ATP binding", "nucleotide binding", "poly $(A)$ RNA binding", "identical protein binding", "RNA binding", "enzyme binding", "microtubule binding", "hydrolase activity, acting on glycosyl bonds", "calcium-dependent protein binding", and "electron carrier activity" (Figure 1). 


\section{PPI network and Module analysis}

We created the PPI networks to identify and analyze the relationships of DGEs at the protein level. The criterion of combined score $>0.7$ was chosen and the PPI network was constructed by using the 553 nodes and 587 interactions. Among these nodes, the top ten genes with highest scores are shown in Table 2. The top two significant modules of AURKA KO versus Control in LP-1 cells were selected to indicate the functional annotation (Figure 2 and Supplemental Table S2).

\section{Reactome Pathway in myeloma cells with AURKA KO}

We identified several signaling pathways by using Reactome Pathway Database (https://reactome.org/). We identified top ten signaling pathways including: "RNA Polymerase I Promoter Opening", "Amyloid fiber formation", "DNA methylation", "ERCC6 (CSB) and EHMT2 (G9a) positively regulate rRNA expression", "Recognition and association of DNA glycosylase with site containing an affected pyrimidine", "PRC2 methylates histones and DNA", "SIRT1 negatively regulates rRNA expression", "Condensation of Prophase Chromosomes", "HDACs deacetylate histones", and "Activated PKN1 stimulates transcription of AR (androgen receptor) regulated genes KLK2 and KLK3" (Supplemental Table S3). We then constructed the reaction map according to the signaling pathways (Figure 3 ).

\section{Potential regulator molecules in AURKA KO cells}

To further know the potential regulator molecules, we introduced the L1000FDW system that can predict and analyze the potential bioactive molecules. The system indicated the potential pathways may be blocked. We selected top ten molecules according to the DEGs and the inhibitor map: "BRD-K53501402", "16,16-dimethylprostaglandin-e2", "cercosporin", "BRD-K10484463", "gemcitabine", "MD-II-051", "BRD-A43640821", “YM155", "BRD-K71935468", and "cymarin" (Supplemental Table S4).

\section{Discussion}


$\mathrm{MM}$ is an accumulation of malignant plasma cells in the bone marrow (BM) that leads to bone lesions and immunodeficiency ${ }^{18}$. Inhibition of Aurora-A kinase gene expression in MM cells by RNAi induces apoptosis and cell death. It also affects G2/M cell-cycle in MM cell lines. Inhibitors of pan-Aurora $(-A$ and $-B)$ and Aurora-B kinases are being tested in clinical trials in patients with $\mathrm{MM}^{19}$. Moreover, MLN8237 is the first oral inhibitor of Aurora-A kinase, which is currently in early-phase clinical testing in patients with $M^{20}$. Thus, our study is based on the AURKA KO in MM cell lines, which may provide the gene evidence for clinical trials of AURKA inhibitors.

To understand the effects of AURKA in the MM cells, we analyzed the LP-1 cells with AURKA KO by the Crispa-Cas9 method. By analyzing the DEGs, we selected 10 proteins that may be important during the development of MM after AURKA KO according to the PPI network analysis. G protein, GPCR and RGS play critical roles in pathophysiology ${ }^{21-23}$ and they are involved in numerous diseases such as cancer ${ }^{24}$, bone dysfunction and inflammatory disease ${ }^{25-29}$. As an important G protein, GNG5 was reported to be increased in gliomas compared with normal samples and it was related to clinicopathologic characteristics ${ }^{30}$. Ube2D1 regulates the ubiquitination of the E3 ubiquitin ligase March-I, which is an independent unfavorable prognostic indicator in lung adenocarcinoma ${ }^{31}$. BUB1B enhances the hepatocellular carcinoma development via triggering the mTORC1 signaling pathway ${ }^{32}$. The ANAPC is an E3 ubiquitin ligase that controls chromosome separation and exit from mitosis in various organisms ${ }^{33}$. BUB1 is reported to enhance the proliferation of liver cancer cells by regulating the SMAD2 phosphorylation ${ }^{34}$. KIF18A enhances the proliferation, invasion and metastasis of HCC cells by promoting the cell cycle signaling pathways and the Akt signaling pathways $^{35}$. UBE2M enhances the cell proliferation through the $\beta$-catenin/cyclin D1 in hepatocellular carcinoma ${ }^{36}$. As the novel HLA class II molecules, HLA-DQA2 and HLADQB2 genes were expressed in human Langerhans cells ${ }^{37}$. Therefore, these proteins are related to the cancer and immune responses.

KEGG and $\mathrm{GO}$ anaylses indicated that immune responses play important roles in the progression of MM cells with AURKA KO. The KEGG and GO anaylses showed the "Leukocyte transendothelial migration", "T cell proliferation", and "positive regulation of monocyte chemotactic protein-1 production", which is associated with the functions of 
immune cells. Previous reports showed that AURKA can drive early signaling during Tcell activation ${ }^{38}$. Here, our results proved that AURKA KO in MM can significantly affect the expression and activation of immune cells such as T cells. Moreover, we found that Hippo pathways were involved in the progression of MM under the regulation of AURKA. The Hippo signaling pathway is an important mediator of oncogenesis in solid tumors ${ }^{39}$. Recent finding showed that there was a direct link between the Hippo pathway, NF-kB, and circadian clock activity ${ }^{40}$. NF-KB is related to numerous inflammatory diseases such as $\mathrm{RA}$ and $\mathrm{OA}^{25,41,42}$. Circadian clock genes regulate the majority of physiology and pathophysiology processes, such as metabolism, apoptosis, aging and diseases ${ }^{43-45}$. Yap1-mediated NF-кB activity interrupts the circadian oscillation by inhibiting Per1, Per2, and Cry2 levels ${ }^{40}$. Adrian Rivera-Reyes also found the inhibition of the UPR and clock caused by YAP1 support a shift in metabolism toward cancer cell-associated glycolysis and hyper-proliferation ${ }^{40}$. Another interesting finding is that the "Alzheimer's disease" and "Influenza A" were changed during the AURKA KO in MM, which suggested that the treatment of MM by targeting the AURKA may affect the therapy effects of these two diseases.

Briefly, we identified the potential biomarkers and pathways for MM cells during AURKA KO. Immune dysfunction and cancer pathways are two key processes during the treatment of AURKA KO. Future studies will focus on the administration of potential AURKA inhibitors on clinical trials. This study thus provides further insights into the treatment of MM under inhibiting AURKA, which may facilitate the drug development. 


\section{Reference}

[1] Kumar SK, Rajkumar V, Kyle RA, van Duin M, Sonneveld P, Mateos MV, Gay F, Anderson KC: Multiple myeloma. Nat Rev Dis Primers 2017, 3:17046.

[2] Fairfield H, Falank C, Avery L, Reagan MR: Multiple myeloma in the marrow: pathogenesis and treatments. Ann N Y Acad Sci 2016, 1364:32-51.

[3] Rajkumar SV: Multiple myeloma. Curr Probl Cancer 2009, 33:7-64.

[4] Gerecke C, Fuhrmann S, Strifler S, Schmidt-Hieber M, Einsele H, Knop S: The Diagnosis and Treatment of Multiple Myeloma. Dtsch Arztebl Int 2016, 113:470-6.

[5] Balakumaran A, Robey PG, Fedarko N, Landgren O: Bone marrow microenvironment in myelomagenesis: its potential role in early diagnosis. Expert Rev Mol Diagn 2010, 10:465-80.

[6] Tai YT, Anderson KC: Targeting B-cell maturation antigen in multiple myeloma. Immunotherapy 2015, 7:1187-99.

[7] Ding L, Cao J, Lin W, Chen H, Xiong X, Ao H, Yu M, Lin J, Cui Q: The Roles of Cyclin-Dependent Kinases in Cell-Cycle Progression and Therapeutic Strategies in Human Breast Cancer. Int J Mol Sci 2020, 21.

[8] Fu J, Bian M, Jiang Q, Zhang C: Roles of Aurora kinases in mitosis and tumorigenesis. Mol Cancer Res 2007, 5:1-10.

[9] Willems E, Dedobbeleer M, Digregorio M, Lombard A, Lumapat PN, Rogister B: The functional diversity of Aurora kinases: a comprehensive review. Cell Div 2018, 13:7.

[10] Kamran M, Long ZJ, Xu D, Lv SS, Liu B, Wang CL, Xu J, Lam EW, Liu Q: Aurora kinase $A$ regulates Survivin stability through targeting FBXL7 in gastric cancer drug resistance and prognosis. Oncogenesis 2017, 6:e298.

[11] Dutta-Simmons J, Zhang Y, Gorgun G, Gatt M, Mani M, Hideshima T, Takada K, Carlson NE, Carrasco DE, Tai YT, Raje N, Letai AG, Anderson KC, Carrasco DR: Aurora kinase $A$ is a target of $\mathrm{Wnt} /$ beta-catenin involved in multiple myeloma disease progression. Blood 2009, 114:2699-708.

[12] Evans R, Naber C, Steffler T, Checkland T, Keats J, Maxwell C, Perry T, Chau H, Belch A, Pilarski L, Reiman T: Aurora A kinase RNAi and small molecule inhibition of Aurora kinases with VE-465 induce apoptotic death in multiple myeloma cells. Leuk Lymphoma 2008, 49:559-69.

[13] Gu H, Yuan G: Identification of key genes in SARS-CoV-2 patients on bioinformatics analysis. bioRxiv 2020:2020.08.09.243444.

[14] Gu H, Yuan G: Identification of potential biomarkers and inhibitors for SARS-CoV-2 infection. medRxiv 2020:2020.09.15.20195487.

[15] Gu H, Yuan G: Identification of potential key genes for SARS-CoV-2 infected human bronchial organoids based on bioinformatics analysis. bioRxiv 2020:2020.08.18.256735.

[16] Gu H, Yuan G: Identification of specific biomarkers and pathways in the synovial tissues of patients with osteoarthritis in comparison to rheumatoid arthritis. bioRxiv 2020:2020.10.22.340232.

[17] Hanming G, Wei W, Gongsheng Y: Research Square 2020.

[18] Oranger A, Carbone C, Izzo M, Grano M: Cellular mechanisms of multiple myeloma bone disease. Clin Dev Immunol 2013, 2013:289458.

[19] Santo L, Hideshima T, Cirstea D, Bandi M, Nelson EA, Gorgun G, Rodig S, Vallet S, Pozzi S, Patel K, Unitt C, Squires M, Hu Y, Chauhan D, Mahindra A, Munshi NC, 
Anderson KC, Raje N: Antimyeloma activity of a multitargeted kinase inhibitor, AT9283, via potent Aurora kinase and STAT3 inhibition either alone or in combination with lenalidomide. Clin Cancer Res 2011, 17:3259-71.

[20] Kelly KR, Shea TC, Goy A, Berdeja JG, Reeder CB, McDonagh KT, Zhou X, Danaee H, Liu H, Ecsedy JA, Niu H, Benaim E, lyer SP: Phase I study of MLN8237-investigational Aurora A kinase inhibitor--in relapsed/refractory multiple myeloma, nonHodgkin lymphoma and chronic lymphocytic leukemia. Invest New Drugs 2014, 32:48999.

[21] Yuan G, Yang S, Liu M, Yang S: RGS12 is required for the maintenance of mitochondrial function during skeletal development. Cell Discov 2020, 6:59.

[22] Yuan G, Yang S, Yang S, Ng A, Oursler MJ: RGS12 is a critical proinflammatory factor in the pathogenesis of inflammatory arthritis via acting in Cox2-RGS12-NF kappa B pathway activation loop. J Bone Miner Res: WILEY 111 RIVER ST, HOBOKEN 07030-5774, NJ USA, 2019. pp. 147-.

[23] Zhu Z, Hua B, Xu L, Yuan G, Li E, Li X, Sun N, Yan Z, Lu C, Qian R: CLOCK promotes 3T3-L1 cell proliferation via Wnt signaling. IUBMB Life 2016, 68:557-68.

[24] Fu C, Yuan G, Yang ST, Zhang D, Yang S: RGS12 Represses Oral Cancer via the Phosphorylation and SUMOylation of PTEN. J Dent Res 2020:22034520972095.

[25] Yuan G, Yang S, Ng A, Fu C, Oursler MJ, Xing L, Yang S: RGS12 Is a Novel Critical NF-kappaB Activator in Inflammatory Arthritis. iScience 2020, 23:101172.

[26] Yuan G, Yang S, Gautam M, Luo W, Yang S: Macrophage regulator of G-protein signaling 12 contributes to inflammatory pain hypersensitivity. Annals of Translational Medicine 2021.

[27] Zhu Z, Xu L, Cai T, Yuan G, Sun N, Lu C, Qian R: Clock represses preadipocytes adipogenesis via GILZ. J Cell Physiol 2018, 233:6028-40.

[28] Cai T, Hua B, Luo D, Xu L, Cheng Q, Yuan G, Yan Z, Sun N, Hua L, Lu C: The circadian protein CLOCK regulates cell metabolism via the mitochondrial carrier SLC25A10. Biochim Biophys Acta Mol Cell Res 2019, 1866:1310-21.

[29] Mao SZ, Fan XF, Xue F, Chen R, Chen XY, Yuan GS, Hu LG, Liu SF, Gong YS: Intermedin modulates hypoxic pulmonary vascular remodeling by inhibiting pulmonary artery smooth muscle cell proliferation. Pulm Pharmacol Ther 2014, 27:1-9.

[30] Yang B, Han ZY, Wang WJ, Ma YB, Chu SH: GNG5 is an unfavourable independent prognostic indicator of gliomas. J Cell Mol Med 2020, 24:12873-8.

[31] Liu J, Cheng Y, Zheng M, Yuan B, Wang Z, Li X, Yin J, Ye M, Song Y: Targeting the ubiquitination/deubiquitination process to regulate immune checkpoint pathways. Signal Transduct Target Ther 2021, 6:28.

[32] Ma Q, Liu Y, Shang L, Yu J, Qu Q: The FOXM1/BUB1B signaling pathway is essential for the tumorigenicity and radioresistance of glioblastoma. Oncol Rep 2017, 38:3367-75.

[33] Pesin JA, Orr-Weaver TL: Regulation of APC/C activators in mitosis and meiosis. Annu Rev Cell Dev Biol 2008, 24:475-99.

[34] Zhu LJ, Pan Y, Chen XY, Hou PF: BUB1 promotes proliferation of liver cancer cells by activating SMAD2 phosphorylation. Oncol Lett 2020, 19:3506-12.

[35] Luo W, Liao M, Liao Y, Chen X, Huang C, Fan J, Liao W: The role of kinesin KIF18A in the invasion and metastasis of hepatocellular carcinoma. World $\mathrm{J}$ Surg Oncol 2018, 16:36. 
[36] Zhang GC, Yu XN, Sun JL, Xiong J, Yang YJ, Jiang XM, Zhu JM: UBE2M promotes cell proliferation via the beta-catenin/cyclin D1 signaling in hepatocellular carcinoma. Aging 2020, 12:2373-92.

[37] Lenormand C, Bausinger H, Gross F, Signorino-Gelo F, Koch S, Peressin M, Fricker D, Cazenave JP, Bieber T, Hanau D, de la Salle H, Tourne S: HLA-DQA2 and HLA-DQB2 genes are specifically expressed in human Langerhans cells and encode a new HLA class II molecule. J Immunol 2012, 188:3903-11.

[38] Blas-Rus N, Bustos-Moran E, Perez de Castro I, de Carcer G, Borroto A, Camafeita E, Jorge I, Vazquez J, Alarcon B, Malumbres M, Martin-Cofreces NB, Sanchez-Madrid F: Aurora A drives early signalling and vesicle dynamics during T-cell activation. Nat Commun 2016, 7:11389.

[39] Pan D: The hippo signaling pathway in development and cancer. Dev Cell 2010, 19:491-505.

[40] Rivera-Reyes A, Ye S, G EM, Egolf S, G EC, Chor S, Liu Y, Posimo JM, Park PMC, Pak K, Babichev Y, Sostre-Colon J, Tameire F, Leli NM, Koumenis C, D CB, Mancuso A, Weber K, Gladdy R, Qi J, Eisinger-Mathason TSK: YAP1 enhances NF-kappaBdependent and independent effects on clock-mediated unfolded protein responses and autophagy in sarcoma. Cell Death Dis 2018, 9:1108.

[41] Yuan G, Xu L, Cai T, Hua B, Sun N, Yan Z, Lu C, Qian R: Clock mutant promotes osteoarthritis by inhibiting the acetylation of NFkappaB. Osteoarthritis Cartilage 2019, 27:922-31.

[42] Yuan G, Hua B, Cai T, Xu L, Li E, Huang Y, Sun N, Yan Z, Lu C, Qian R: Clock mediates liver senescence by controlling ER stress. Aging 2017, 9:2647-65.

[43] Yuan G, Hua B, Yang Y, Xu L, Cai T, Sun N, Yan Z, Lu C, Qian R: The Circadian Gene Clock Regulates Bone Formation Via PDIA3. J Bone Miner Res 2017, 32:861-71.

[44] Zhu Z, Hua B, Shang Z, Yuan G, Xu L, Li E, Li X, Sun N, Yan Z, Qian R, Lu C: Altered Clock and Lipid Metabolism-Related Genes in Atherosclerotic Mice Kept with Abnormal Lighting Condition. Biomed Res Int 2016, 2016:5438589.

[45] Xu L, Cheng Q, Hua B, Cai T, Lin J, Yuan G, Yan Z, Li X, Sun N, Lu C, Qian R: Circadian gene Clock regulates mitochondrial morphology and functions by posttranscriptional way. bioRxiv 2018:365452. 
bioRxiv preprint doi: https://doi.org/10.1101/2021.03.21.436324; this version posted March 22, 2021. The copyright holder for this preprint (which was not certified by peer review) is the author/funder, who has granted bioRxiv a license to display the preprint in perpetuity. It is made available under aCC-BY-NC-ND 4.0 International license.

\section{Figures and Figure Legends}
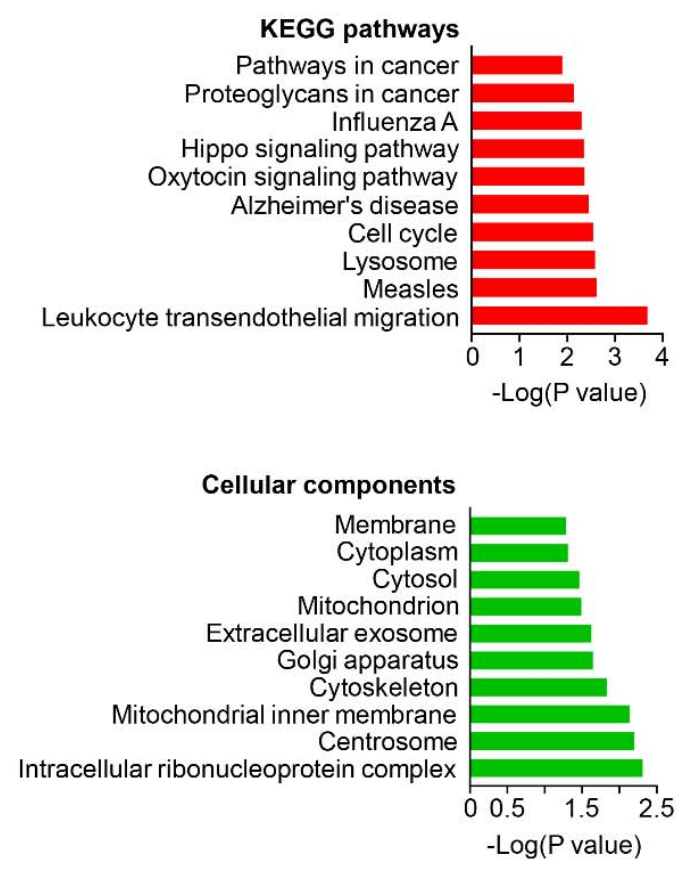

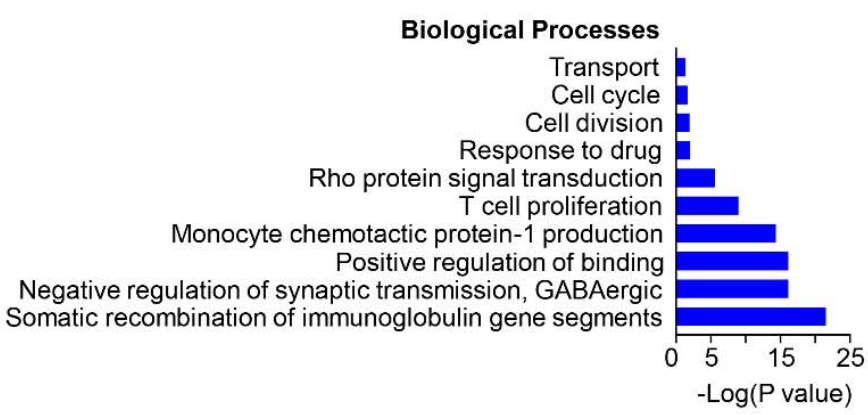

Molecular Functions ATP binding

Nucleotide binding Poly(A) RNA binding Identical protein binding RNA binding Enzyme binding

Microtubule binding Hydrolase activity, acting on glycosyl bonds Calcium-dependent protein binding Electron carrier activity

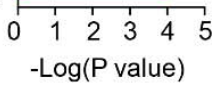

Figure 1. The KEGG pathways, biological process, cellular component, and molecular function terms enriched by the DEGs. DEGs =differentially expressed genes, KEGG = Kyoto Encyclopedia of Genes and Genomes. 


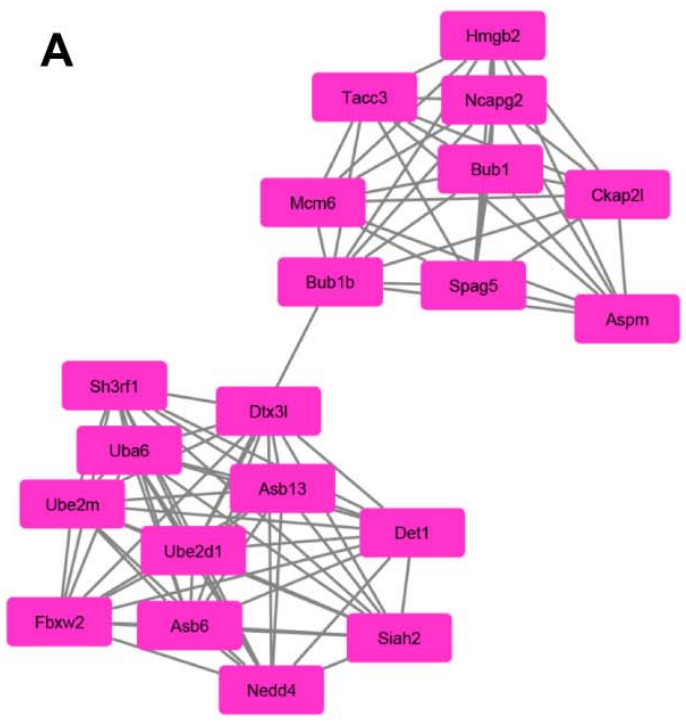

B

Figure 2. Top two modules (A, module 1 and $B$, module 2) from the PPI network of MM cells with AURKA KO. 


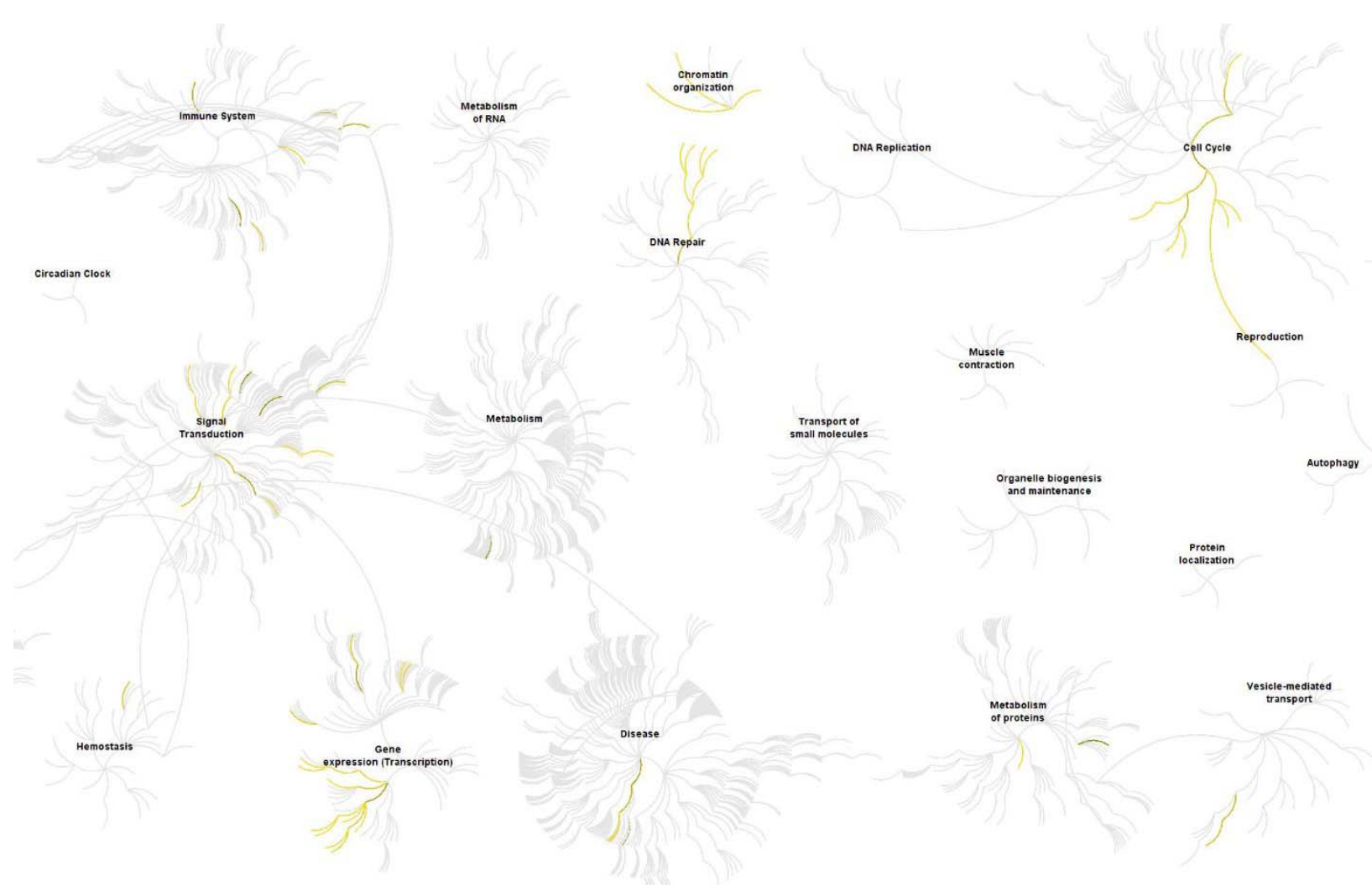

Figure 3. The Reactom pathway visualization map. Input genes are represented by the top significantly changed genes obtained from the GSE163589 dataset $(P<0.01)$. The yellow color represents the most relevant signaling pathways. 


\section{p-value MOA}

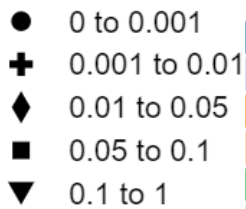

protein synthesis inhibitor NFkB pathway inhibitor topoisomerase inhibitor HDAC inhibitor ATPase inhibitor HMGCR inhibitor PI3K inhibitor tubulin polymerization inhibitor HSP inhibitor mTOR inhibitor adrenergic receptor antagonist MEK inhibitor EGFR inhibitor other unknown retinoid receptor agonist glucocorticoid receptor agonist CDK inhibitor adrenergic receptor agonist dopamine receptor antagonist

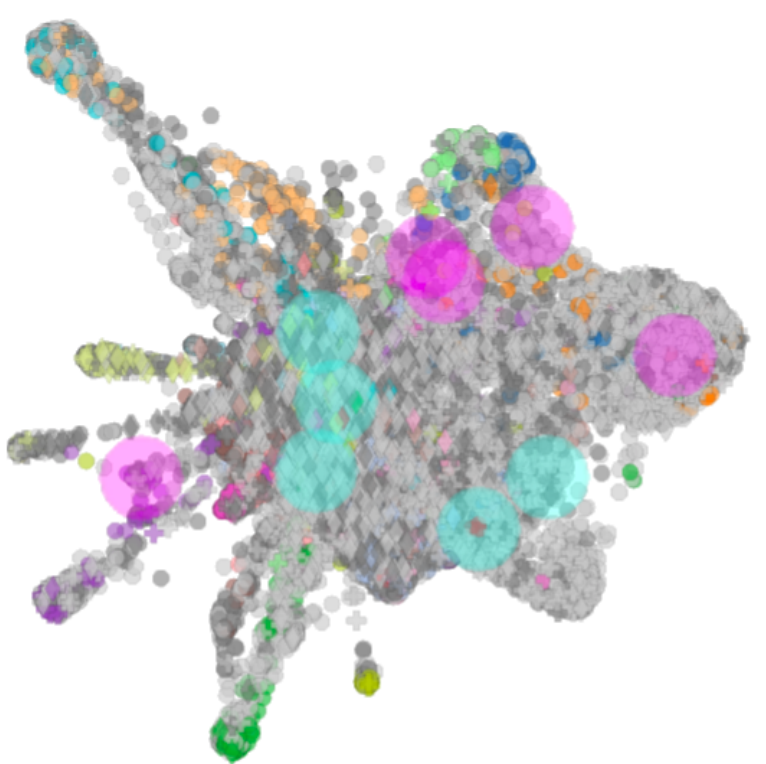

Figure 4. Inhibitor prediction against MM by L1000FDW visualization. Input genes are represented by the significantly changed genes obtained from the GSE163589 dataset. Dots are the Mode of Action (MOA) of the respective drug. 
Table 1

\begin{tabular}{llll}
\hline Entrez gene & Gene Symble & Fold-change & Regulation \\
\hline \multicolumn{1}{l}{ Top 10 down-regulated DEGs } & & \\
79805 & VASH2 & -1.825741858 & Down \\
642597 & AKAIN1 & -1.825741858 & Down \\
55975 & KLHL7 & -1.81897851 & Down \\
10142 & AKAP9 & -1.81323663 & Down \\
26256 & CABYR & -1.81323663 & Down \\
81567 & TXNDC5 & -1.811197356 & Down \\
51303 & FKBP11 & -1.810806906 & Down \\
101928635 & LOC101928635 & -1.80972627 & Down \\
5701 & PSMC2 & -1.808122644 & Down \\
284615 & ANKRD34A & -1.807454814 & Down \\
Top 10 up-regulated DEGs & & \\
346689 & KLRG2 & 1.825741858 & up \\
4145 & MATK & 1.825741858 & up \\
83417 & FCRL4 & 1.825741858 & up \\
3553 & IL1B & 1.825741858 & up \\
3362 & HTR6 & 1.825741858 & up \\
55220 & KLHDC8A & 1.825741858 & up \\
9087 & TMSB4Y & 1.825741858 & up \\
1278 & COL1A2 & 1.825741858 & up \\
10814 & CPLX2 & 1.821415451 & up \\
5126 & PCSK2 & 1.820356178 & up \\
\hline
\end{tabular}


Table 2. Top ten genes demonstrated by connectivity degree in the PPI network

\begin{tabular}{lll}
\hline Gene Symbol & Gene title & Degree \\
\hline GNG5 & G protein subunit gamma 5 & 20 \\
UBE2D1 & ubiquitin conjugating enzyme E2 D1 & 17 \\
BUB1B & BUB1 mitotic checkpoint serine/threonine & 16 \\
& kinase B & \\
ANAPC1 & anaphase promoting complex subunit 1 & 15 \\
BUB1 & BUB1 mitotic checkpoint serine/threonine & 15 \\
& kinase & 14 \\
KIF18A & kinesin family member 18A & 14 \\
UBE2M & ubiquitin conjugating enzyme E2 M \\
HLA-DQA2 & major histocompatibility complex, class II, & 13 \\
& DQ alpha 2 \\
HLA-DQA1 & major histocompatibility complex, class II, & 13 \\
& DQ alpha 1 \\
HLA-DPB1 & major histocompatibility complex, class II, & 13 \\
& DP beta 1 & \\
\hline
\end{tabular}

